\title{
Genomic-based targeted therapy and management of advanced non-small cell lung cancer: Protocol for a qualitative study of oncologists' perceptions and behaviors regarding genomic-based targeted therapy
}

\author{
Guoqing J. Chen ${ }^{1,2}$, J ennifer Arney ${ }^{3,4}$, Ashley Helm5 ${ }^{5}$ Ursula Braun ${ }^{3,5}$, Peter Richardson ${ }^{3,5}$, Ping \\ Chen $^{6}$, Teresa G. Hayes ${ }^{3,5}$ \\ 1. University of Kansas, School of Medicine, Kansas City, Kansas, USA. 2. Department of Veterans Affairs, Veterans Health \\ Administration, Office of Research and Development, Kansas City VA Medical Center, Kansas City, MO, USA. \\ 3. Department of Medicine, Baylor College of Medicine, Houston, Texas, USA. 4. Department of Sociology, University of \\ Houston-Clear Lake, Houston, Texas, USA. 5. Michael E. DeBakey VA Medical Center, Houston, Texas, USA. 6. University \\ of Massachusetts, Boston, Massachusetts, USA.
}

Correspondence: Teresa G. Hayes. Address: Michael E. DeBakey VA Medical Center, Hematology-Oncology Section VA 111H, 2002 Holcombe Blvd., Houston, TX, USA. Email: thayes@bcm.edu

Received: December 1, 2014

DOI : $10.5430 /$ jst.v5n1p33

\begin{abstract}
Background: Pilot data revealed gaps in knowledge and utilization of genomic-based targeted therapy among oncologists. We report these findings, and offer a protocol for a qualitative research project aimed to elicit oncologists' perceptions regarding use of genomic-based targeted therapy for Veterans with lung cancer at the VHA. Using the Cabana et al. theoretical framework, a model of behavior change among providers, the study will identify how oncologists' perceptions and facility characteristics potentially affect implementation of genomic-based targeted therapy in routine care.

Methods and results: We will conduct a minimum of 40 and up to 60 semi-structured interviews with oncologists to elicit perceptions of genomic-based targeted therapy in four domains: 1) knowledge; 2) attitudes; 3) intent; and 4) barriers and facilitators for practicing genomic-based targeted therapy.

Conclusion: This study is the first to examine decision-making surrounding the use of genomic based targeted therapy in lung cancer management. Findings will lay the foundation to design and implement effective strategies centering on integrating genomic medicine with routine practice, and will shed light on the decision-making processes of oncologists regarding selection of genomic-based and non-genomic-based treatment plans in managing lung cancer in veterans. This research is part of a larger study that will examine the utilization, clinical effectiveness, and cost effectiveness of genomicbased therapies in the treatment of advanced lung cancer.
\end{abstract}

\section{Key words}

Lung Cancer, Genomic therapy, Utilization, Mixed methods research, Comparative effectiveness research, Cost effectiveness research 


\section{Introduction}

Lung cancer is the second most common cancer in the United States, with an estimated 221,200 new cases in $2015^{[1]}$. It is also the leading cause of cancer-related mortality in the U.S., with approximately 158,040 deaths expected in that same year. Most lung cancers are one of two types: 1) small cell lung cancer (SCLC), comprising 15\% of diagnoses; and 2) non-small cell lung cancer (NSCLC), comprising the remaining 85\%. Although screening for lung cancer with low dose CT scans can be effective, it is expensive and not yet widely used in practice. As a result, lung cancer is difficult to detect at an early stage, with approximately $75 \%$ of diagnosed patients having advanced disease (stage IIIB or IV) at the time of diagnosis. The 5-year survival rate for all stages is about $15 \%$. The treatment is dependent upon the cancer location, stage and type, and patient health status. The usual non-genomic-based treatment options include surgery, radiation therapy, chemotherapy, and angiogenesis inhibitors, but more recently, genomic-based therapies have emerged.

Genomic-based targeted therapy (GBTT) has emerged as an alternative option in clinical management of NSCLC. Erlotinib, the tyrosine kinase inhibitor (TKI) of the epidermal growth factor receptor (EGFR) is used in lung cancer management. In August 2011, the Food and Drug Administration (FDA) approved crizotinib, another class of genomicbased targeted therapy, for the treatment of patients with locally advanced or metastatic NSCLC that is positive for the anaplastic lymphoma kinase (ALK) mutation, an EML4-ALK fusion oncogene. The use of erlotinib and crizotinib have been recommended in the National Comprehensive Cancer Network (NCCN) clinical practice guidelines for advanced lung cancer with the appropriate gene mutations ${ }^{[2]}$. Neither erlotinib nor crizotinib are listed in the VA drug formulary and they require special permission for VA oncologists to use them to treat lung cancer.

The purpose of this study is to identify factors that affect oncologists' decisions regarding use of genomic-based targeted therapy, including erlotinib and crizotinib, and genomic testing, for patients with lung cancer at the VHA. We will conduct semi-structured interviews with oncologists to elicit information in four domains: 1) knowledge; 2) attitudes; and 3) intent; and 4) barriers and facilitators for practicing genomic-based targeted therapy. In addition, we will collect pilot data regarding oncologists’ decision making regarding palliative care for lung cancer patients. Information from this study will help us understand the decision-making processes of oncologists regarding selection of genomic-based and non-genomicbased treatment plans in managing lung cancer in veterans. Findings will lay the foundation to design and implement effective strategies centering on integrating genomic medicine with routine practice. This aim is one part of a larger study that will examine the utilization, clinical effectiveness and cost effectiveness of genomic-based therapy in the management of NSCLC at the VHA.

\subsection{Factors potentially associated with use of genomic-based targeted therapy}

Physicians play a key role for patients in terms of access to genomic-based targeted treatment. Although there is no study examining physician practice patterns in ordering EGFR and ALK mutation tests and prescribing in routine practice settings, physicians' knowledge, attitudes, beliefs, and specialization may affect decisions to provide genomic medicine ${ }^{[3-7]}$. Other factors affecting physicians’ ordering behaviors have been documented, including physicians' age and gender, experience, financial incentives, awareness of the cost of testing, fear of a malpractice lawsuit, and practice setting ${ }^{[8-14]}$. Older physicians were likely to order more tests, while younger physicians were likely to adhere to the recommended practice guidelines ${ }^{[9,15]}$. Specialists were reported to order more tests than primary care physicians ${ }^{[11,15,16]}$. Academic physicians ordered more tests than non-academic physicians ${ }^{[17]}$. Increased physician experience, knowledge, and beliefs were also associated with an increase or decrease in ordering tests ${ }^{[18,19]}$. Additionally, physicians' personal beliefs, which are not evidenced-based, affect test ordering. Physicians who believed that cancer screening reduces

cancer-related mortality ordered the cancer tests more often than those who did not ${ }^{[20]}$. Physicians who were not convinced by clinical trial results were less likely to practice guideline-recommended care. 


\subsection{Previous research on providers' views on using genomic services at the VHA}

Previous research examined VHA providers' intention toward the utilization of genomic services at the VHA through the lens of the Theory of Planned Behavior (TPB) ${ }^{[21]}$. Semi-structured interviews were conducted with 20 providers working in different units at the South Texas Veterans Health Care System (STVHCS). The interviews focused on assessing providers' behavioral, normative, and control beliefs regarding the delivery of genomic medicine at the STVHCS. Findings indicated that all participating providers perceived genomic medicine to be an important area in medicine. They agreed that the VHA has the necessary infrastructure to foster the delivery of genomic services. The majority of participants (90\%) agreed that primary care providers will play a major role in delivering genomic services. Providers indicated that their peers' opinions about genomic services may affect their decisions about utilizing genomic services. However, most providers (85\%) raised concerns about the impact of using genomic services on the process of care, such as adding additional demands on busy clinical practices. Participants indicated that decision support tools may facilitate the implementation of genomic services into VHA clinical practice.

In light of the many gaps in the literature regarding the utilization and perceptions of GBTT and provider factors that affect the use of GBTT, we propose to conduct a study aimed to elicit oncologists' knowledge, attitudes, intentions, and perceived barriers and facilitators regarding use of genomic-based targeted therapy for Veterans with lung cancer at the VHA. Using a model of behavior change among providers, we will describe how oncologists' perceptions and facility characteristics potentially affect adoption of genomic-based targeted therapy in routine care. In addition, the team will collect pilot data for a future project on decisions regarding palliative care for lung cancer patients. Pilot interviews with 10 oncology fellows at one of the largest VA medical centers in the Southwest found gaps in knowledge that should be explored further. We propose the following study to better evaluate the magnitude of the gaps in knowledge and to examine other areas of uncertainty at a national level.

\section{Methods}

\subsection{Design overview}

Interviews will elicit oncologists' perceptions of genomic-based targeted therapy for Veterans with lung cancer at the VHA. Using constructs derived from Cabana et al.’s theoretical framework, we will investigate oncologists' views on using genomic-based targeted therapy for patients with lung cancer ${ }^{[22]}$. Cabana and colleagues specify that physicians' lack of adherence to practice guidelines is shaped by a variety of barriers involving knowledge and attitudes, including lack of familiarity, lack of awareness, lack of agreement with guidelines, lack of outcome expectancy, lack of self-efficacy, and lack of motivation. Additionally, actual behavior change can be shaped by external and environmental barriers, such as patient factors, guideline factors, time, resources, and organizational constraints. Accordingly, our semi-structured interviews will elicit information on four domains: 1) knowledge, 2) attitudes, 3) intent to use genomicbased targeted therapy, and 4) barriers/facilitators for practicing genomic-based targeted therapy. The interviews will also collect pilot data regarding palliative care for lung cancer patients.

\subsection{Participants}

We will compile a list of medical oncologists at the VHA. To select our subjects, we will first stratify the provider list by comprehensive cancer center status (yes/no). To select our interview participants we will use purposive sampling. We will contact ten potential participants from each list (comprehensive and non-comprehensive cancer center status) in waves, until we reach saturation. We will enroll a minimum 40 and no more than 60 medical oncologists for telephone interviews.

The PI of this study will send email invitations for medical oncologists who treat patients with lung cancer at the VHA and invite them to participate in a one-time semi-structured interview. If potential oncologists do not reply to the first 
invitation, the research assistant will follow-up with a second email invitation 5 days after the initial invitation is sent. Oncologists will be contacted no more than three times to be invited to participate in our study. Verbal consent will be obtained from oncologists who express interest in participating in our study. Their interviews will then be scheduled with accommodations outside of clinic hours.

\subsection{Data collection}

We conducted 10 pilot interviews with oncology fellows to refine the semi structured interview guide (see Table 1). The refined guide will be used to conduct telephone interviews with up to 60 oncologists at the VHA. All interviews will be audio-recorded with the participant's consent. Interview recordings will be sent for transcription the day after the interview; we will begin analyzing transcripts per our data analysis strategy as soon as they are received.

Table 1. Oncologist Interview Guide

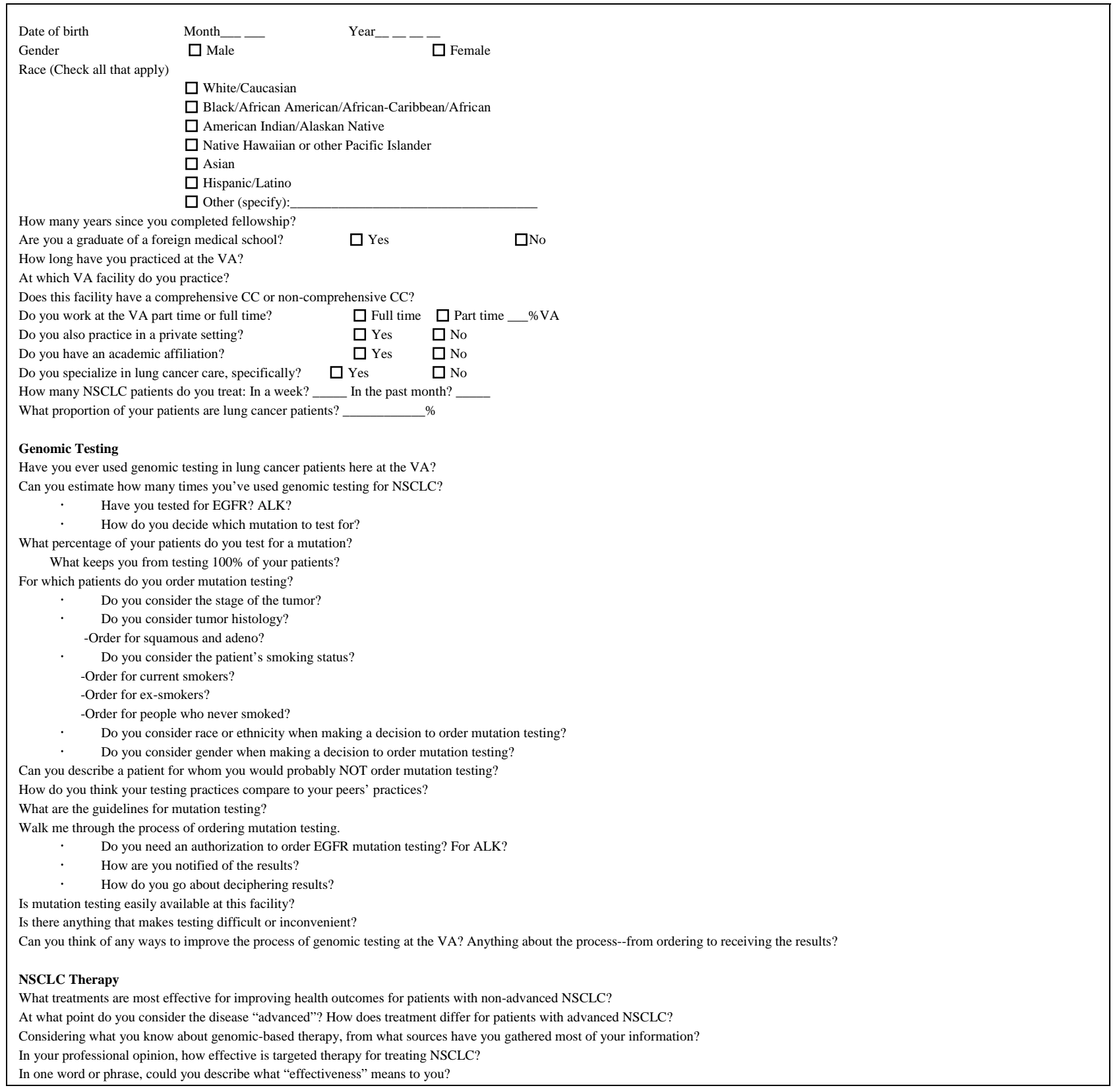


Table 1. (continued.)

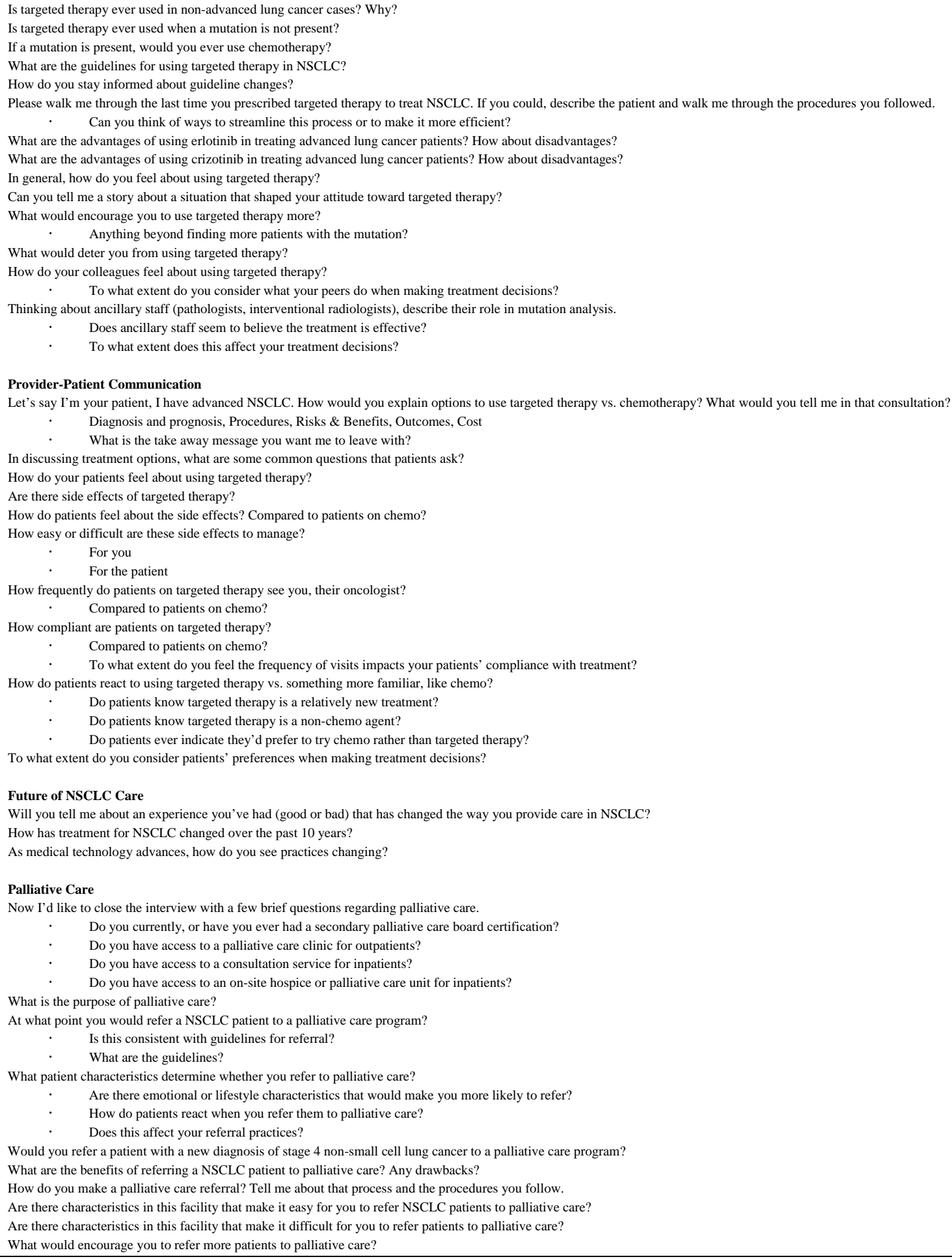

\subsection{Sample size}

Sample size will be determined based on the concept of saturation. As new codes indicate new concepts of importance, a lack of new codes is indicative that no new information would be generated by additional interviews. To minimize participant burden, interviews will be scheduled and analyzed concurrently until saturation of information is reached. For this analysis, we will consider our data saturated when three consecutive transcripts render no new codes. Because we 
hope to make meaningful comparisons between comprehensive and non-comprehensive cancer facilities, we will ensure that we reach saturation in both groups of transcripts.

\subsection{Analysis}

We will perform qualitative content analysis using principles of grounded theory ${ }^{[23]}$. The analysis from the semistructured interviews will include identification of broad categories related to: 1) knowledge, 2) attitudes, 3) intent to use genomic-based targeted therapy, and 4) facilitators and barriers to using genomic-based targeted therapy ${ }^{[21]}$.

Analysis of the qualitative data will begin during data collection to inform subsequent semi-structured interviews ${ }^{[23]}$. Transcribed interviews will be analyzed using techniques adapted from grounded theory analysis. Two independent coders will analyze all interview data. To develop an initial code list, the first coder will identify, name, categorize, and describe phenomena found in the first five transcripts. When the initial code list has been compiled, the team will meet to discuss and refine the code list. The coding scheme will be applied to the remaining transcripts. To establish inter-coder reliability and maximize validity and reliability of the coding scheme, the second coder will examine each transcript and the codes, and will confirm, deny, or add codes as necessary. The two coders will meet after every 10 transcripts are coded to discuss and resolve any discrepancies, and to discuss added codes. Any unresolved coding discrepancies will be resolved through group discussion. Finally, we will distinguish salient themes in the data, and compare and contrast participants' responses to identify meaningful associations and patterns in the data.

Charting will be used to organize the data and compare and contrast providers' responses; this information will be used to interpret the data by identifying meaningful patterns that best explain utilization of genomic-based targeted therapy in the management of Veterans diagnosed with NSCLC within the VA healthcare system. To ensure a high quality of qualitative data, we will use triangulation and subject "member" checking approaches. Findings from the qualitative data will be nested within the quantitative findings and will provide important context to understand providers' knowledge, attitudes, intent to use genomic-based targeted therapy, facilitators and barriers, and patterns of utilization. Using both qualitative and quantitative data will contribute to the validity of the results through triangulation. The study was approved by the local institutional review board (IRB).

\section{Pilot data results and discussion}

Genomic-based anticancer agents such as EGFR TKIs have been emerging as first-line treatment for NSCLC. Clinical evidence shows that in unselected patients with advanced NSCLC, a subset of the patients ( $10 \%$ to $20 \%$ ) have a favorable response to treatment, whereas in patients with EGFR mutations the response rates are much higher, up to 94\%. Clinical practice guidelines of the NCCN and the American Society of Clinical Oncology (ASCO) recommend using EGFR-TKIs (erlotinib) as first-line therapy for NSCLC patients with EGFR mutation positive lung cancer ${ }^{[2]}$.

Lung cancer is the leading cause of cancer-related death in the U.S general population as well as in the Veteran population, and NSCLC is the most common type of lung cancer, accounting for approximately $85 \%$ of all lung cancer cases ${ }^{[1]}$. Prior to our understanding of NSCLC molecular tumor biology, the conventional treatment for advanced NSCLC was to use systemic chemotherapy that kills cancerous cells but also has toxic effects on normal cells. Due to the high level of toxicity of chemotherapeutic agents, many patients with poor health status do not tolerate this treatment. Genomic-based targeted therapy is not inexpensive. To our knowledge, there are few studies that assess the evidence-based clinical effectiveness and values of using genomic-based targeted therapy relative to conventional therapies for lung cancer patients in VA. This qualitative aim, in conjunction with the larger quantitative study, seeks to fill this gap by assessing the utilization and comparative value (clinical effectiveness and cost-effectiveness), and oncologists' views of delivering genomic-based targeted therapy for Veterans with advanced lung cancer in the VA practice setting. Coding of 10 pilot interviews resulted in four themes consistent with the model specified by Cabana and colleagues ${ }^{[22]}$. These themes allowed for the classify- 
cation of respondents' perceptions into four categories of interest: knowledge, attitudes, intent, and facilitators and barriers to use.

\subsection{Knowledge}

Analysis of the pilot interviews reflected varying degrees of knowledge regarding genomic testing and therapy, though the majority of participants reported they referred to the guidelines to determine which patients to test and treat. Participants identified tumor characteristics and patient characteristics that inform their decisions to request mutation analysis and prescribe GBTT. Tumor characteristics that were cited as justification for ordering testing include tumor histology and stage; however, there was some discrepancy in how stage was defined, which warrants further investigation. Consistent with guidelines, participants stated they prescribe erlotinib when patients are found to be EGFR mutation positive and they prescribe crizotinib when patients are found to have ALK rearrangement. About half of participants, however, discussed cases in their daily practice in which they have prescribed or would prescribe GBTT when no mutation was present.

Patient characteristics that were said to prompt ordering testing and treatment with GBTT included patient demographics such as Asian race, female sex, and non-smoker status, although the majority of participants acknowledged that the guidelines recommended testing for all patients. In the pilot interviews, smoking status emerged as an important patient characteristic that influenced oncologists' decisions to test for mutations. Therefore, more questions were added to the interview guide to better tease out nuances regarding testing for smokers vs. non-smokers. A minority of participants indicated that they would use GBTT in patients without mutation for a variety of reasons including poor performance status, inability to tolerate chemo, or the inability for the patient to appear at the facility for chemo treatment. Two participants mentioned that GBTT, in particular crizotinib, may not be safe for patients with poor vision and/or liver disease.

The pilot interviews revealed that there were gaps in participants' knowledge regarding guidelines, mutation testing, and treatment for NSCLC. In terms of guidelines, about $1 / 3$ of the participants acknowledged uncertainty about guidelines involving use of GBTT in second or third line therapy or contraindications for using GBTT. About 1/3 of the participants were uncertain about policies and procedures for ordering mutation testing at the VA. In all, gaps in knowledge were identified among a minority of participant interviews. It appears that these discrepancies may result from a lack of clear understanding of the guidelines, a lack of experience treating NSCLC patients, or a lack of experience at the VA hospital (see Table 2).

Table 2. Quotations illustrating knowledge of genomic testing and treatment

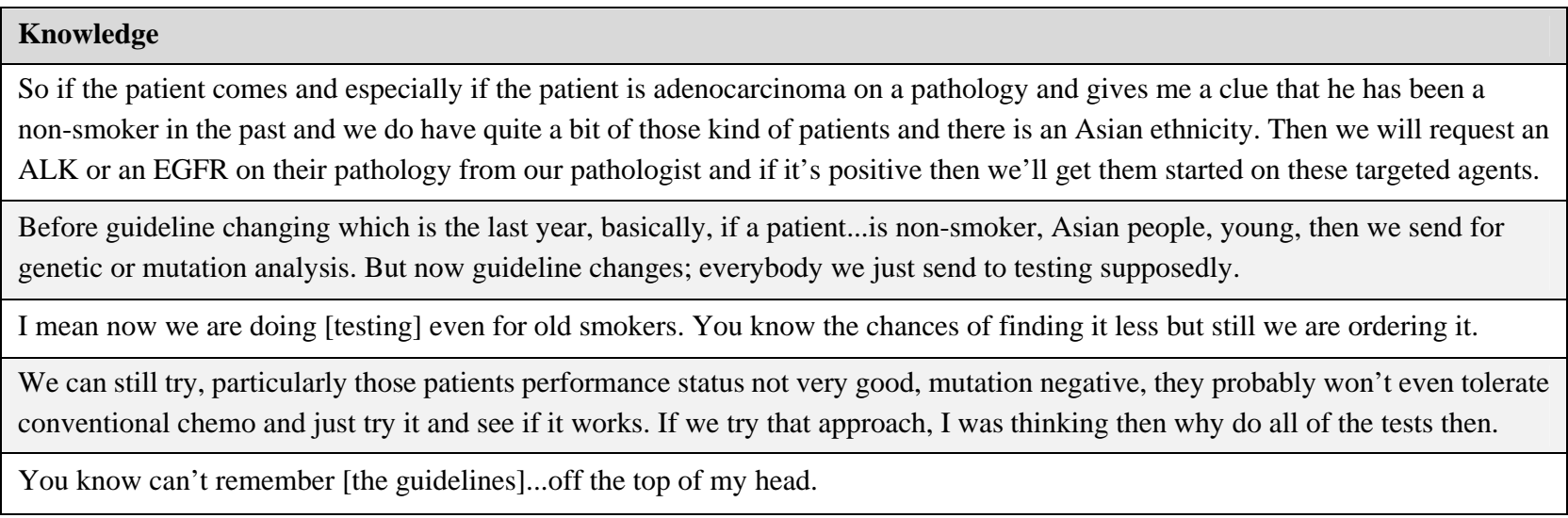

\subsection{Attitudes toward GBTT}

Participants' attitudes toward GBTT were consistently positive. All were supportive of using this type of therapy for advanced NSCLC patients. The most common advantages that were cited involve tolerability, efficacy, and convenience 
for the patient. All pilot participants described GBTT as easily tolerated and generally effective for treating NSCLC, however, two participants indicated some uncertainty regarding the effectiveness of GBTT. Another perceived advantage of GBET is convenience because it requires fewer trips to the hospital for treatment. Just over half of respondents listed side effects such as rash, diarrhea, and liver enzyme dysfunction as a potential drawback to using GBTT. However, almost all of these respondents described these side effects as mild and/or indicated that they can be clinically managed. Interviews revealed other potential drawbacks that may shape prescribing decisions such as a slower response time, resistance to targeted therapy, more frequent treatments, and higher cost compared to chemo therapy.

When asked about colleagues' attitudes toward GBTT, participants indicated that their peers' attitudes were positive. There were no instances in which respondents perceived their colleagues as holding negative attitudes toward prescribing GBTT. When asked about the attitudes of ancillary staff, respondents offered a variety of responses. Most participants reported that they simply did not know how ancillary staff feels about GBTT (see Table 3).

Table 3. Quotations illustrating attitudes toward genomic-based targeted therapy

\begin{tabular}{|c|}
\hline Attitudes toward GBTT \\
\hline $\begin{array}{l}\text { Targeted therapies... are more easily tolerated than chemotherapies for the most part. We choose them based on whether somebody } \\
\text { has a genomic mutation or not and usually they can be done concurrently with some chemotherapies as well. }\end{array}$ \\
\hline $\begin{array}{l}\text { It's better tolerated; it's easier on the patient. It's less trips to the hospital and unless you have severe side effects from the erlotinib } \\
\text { itself, it's easier because it's oral medicine. }\end{array}$ \\
\hline It's a preferred treatment and there's less side effects with it. \\
\hline $\begin{array}{l}\text { It's just a pill a day if they're not having any major side effects. It's just a pill a day but they're able to function better, they're able to } \\
\text { do the things they weren’t able to do before. Like ten years ago it- it was a different disease altogether. }\end{array}$ \\
\hline $\begin{array}{l}\text { You get mild side effects with erlotinib. I mean you get rash and sometimes diarrhea. Some patients get it more severely in which case } \\
\text { you wouldn't want to use it for them. }\end{array}$ \\
\hline $\begin{array}{l}\text { I don't think a lot is going to change my mind although it did take me a while to come into this kind of feeling. It's not just a snapshot; } \\
\text { it's kind of a progression in a year or two. Because from my experience when I see patients who really benefit a lot from it and-and my } \\
\text { own experience kind of bias as well. I have had patients who even benefited more than what the literature show so that will- kind of a } \\
\text { personal bias as well. }\end{array}$ \\
\hline
\end{tabular}

\subsection{I ntent to use GBTT}

Interviews point to overwhelming support for mutation testing and use of GBTT for patients with associated mutations. All participants were supportive of mutation testing and GBTT. In terms of intention to request mutation analysis, the majority of the participants acknowledged that they test all comers with advanced adenocarcinoma. Other participants indicated that they focus testing efforts on a percentage of their adenocarcinoma patients, according to likelihood of mutation. One participant suggested that prior to guideline changes, it was the norm to focus testing on likely candidates (Asian, female, non-smoker); however, it is now the standard of care to test all adenocarcinoma patients. When asked about intent to prescribe GBTT, all participants were very supportive of this type of treatment. All participants were comfortable with the frequency with which they prescribe GBTT, and most participants acknowledged that they would prescribe it more often if more patients were found to have the mutation (see Table 4).

Table 4. Quotations illustrating intent to use genomic-based targeted therapy

\section{Intent to use GBTT}

We are very interested because it totally changes our management and you cannot imagine how beautifully people do on these oral targeted agents so why not you know.

If there are mutations I'll always try my best to go for the p.o. medications. 


\subsection{Barriers and facilitators to genomic testing}

The most commonly mentioned barriers to testing include insufficient tissue sample to conduct the mutation analysis, cost of conducting the test, and inconvenience of ordering the test. Roughly 1/3 of the participants indicated that sometimes patients undergo biopsy, only to find that the tissue sample is too small to conduct the mutation analysis. Since this was a recurring theme in the data, this raises concern that some GBTT candidates may be missed due to this barrier. Interestingly, while about half of the participants suggested that cost of testing could be a barrier, only one participant identified VA insurance as a facilitator to testing.

According to the pilot interviews, the ease of ordering the test, the ease of deciphering results, and VA insurance coverage were cited as facilitators to mutation testing. Requesting the mutation analysis from the pathologist via email was mentioned as a barrier to testing by at least one participant, however, the ability to contact a pathologist via email was listed as a facilitator to testing by about a third of pilot participants. Roughly $1 / 3$ of the participants indicated that ease of deciphering results is a facilitator to their testing. The most common recommendation from participants to facilitate testing was automatic mutation analysis for all adenocarcinoma patients. Another suggested facilitator involved administering testing in the office using a skin sample or buccal swab (see Table 5).

Table 5. Quotations illustrating barriers and facilitators to genomic testing

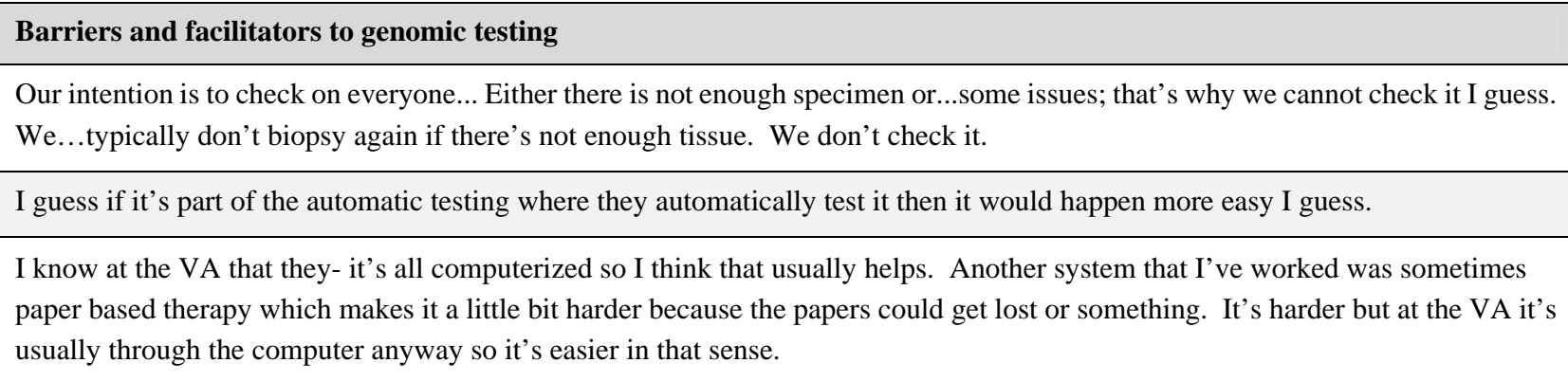

We just shoot an e-mail to our pathologists and they're more than happy to do it. They just need to know that we want it and they're very good at following up with us.

I think it's expensive; I don't know the cost though.

\subsection{Barriers and facilitators to prescribing}

The most common barrier to prescribing targeted therapy was delayed test results, which was identified as a barrier by half of the participants. It is unclear whether a two-week window in testing is a modifiable barrier. Cost was, again, identified as a potential barrier to prescribing GBTT, however, this was mentioned by a minority of participants. The most common facilitator to prescribing GBTT that was cited was finding more mutations. Also mentioned as a facilitator to prescribing, was VA insurance coverage. One participant recommended notifying the primary physician of the mutation analysis test results to facilitate prescribing GBTT. This is also related to the delay in testing that was discussed by a number of participants (see Table 6).

Table 6. Quotations illustrating barriers and facilitators to prescribing genomic therapy

\begin{tabular}{|l|}
\hline Barriers and facilitators to prescribing \\
\hline I think right now it's pretty easy and there's no barrier. \\
\hline If the patient has not a very good insurance and...cannot afford expenses. \\
\hline
\end{tabular}

In this pilot study, we used qualitative interviews to identify gaps in knowledge, perceived disadvantages, and barriers to use regarding GBTT. We expect to find other gaps in knowledge, perceived disadvantages, and barriers to use at other VA sites; therefore, it is important that we study these topics at a national level to get a more complete view of GBTT use in the 
VA. We believe that the findings from this project will provide useful information to improve the provision of genomic medicine for veterans with advanced lung cancer. The expected results from this study will provide important information to policy decision-makers and practicing physicians. The audience and utilizers of the study findings include medical oncologists, VA health policy decision-makers, and researchers in the field. For physicians, these findings will improve their understanding of the added benefits of genotyping-guided treatment in routine practice. To healthcare planners and policy makers, this study will provide evidence-based information about the impact of genotyping-guided treatment on patient outcomes. To researchers, the study results will provide a basis for future studies in the fields of genomic health services and comparative effectiveness. Based on our pilot data regarding palliative care for lung cancer patients, we intend to conduct a thorough examination of oncologists' behavior and decision making process regarding palliative care.

\section{Conclusions}

The proposed research is the first to examine provider factors that affect the use of GBTT in patients with lung cancer. This study will provide valuable information about oncologists' knowledge, attitudes, intentions, and perceived barriers and facilitators in terms of the use of genomic-based targeted therapy for Veterans with lung cancer at the VHA. The findings of this qualitative pilot study uncovered factors that influence oncologists' treatment decisions, which can be used to enhance the execution of the full study and provide useful information for future system-wide implementation in practice of personalized medicine.

\section{Acknowledgement}

This material is based upon work supported by the Department of Veterans Affairs, Veterans Health Administration, Health Services Research and Development Service (11K11.H Grant\#: IIR112-15), and in part by the Center for Innovations in Quality, Effectiveness and Safety (\#CIN 13-413), Michael E. DeBakey VA Medical Center, Houston Texas. The views expressed in this article are those of the authors and do not necessarily represent the views of the Department of Veterans Affairs.

\section{References}

[1] Siegel R, Miller KD, Jemal, A. Cancer Statistics, 2015. Ca Cancer J Clin. 2015; 65(1): 5-29. Epub 2015 Jan 5. http://dx.doi.org/10.3322/caac.21254

[2] NCCN clinical practice guidelines in oncology: non-small cell lung cancer. Version 4. 2015.

[3] Metcalfe S, Hurworth R, Newstead J, et al. Needs assessment study of genetics education for general practitioners in Australia. Genet Med. 2002; 4(2): 71-77. PMid:11882783 http://dx.doi.org/10.1097/00125817-200203000-00004

[4] Baars MJ, Henneman L, Ten Kate LP. Deficiency of knowledge of genetics and genetic tests among general practitioners, gynecologists, and pediatricians: a global problem. Genet Med. 2005; 7(9): 605-610. PMid:16301861 http://dx.doi.org/10.1097/01.gim.0000182895.28432.c7

[5] Freedman AN, Wideroff L, Olson L, et al. US physicians' attitudes toward genetic testing for cancer susceptibility. Am J Med Genet A. 2003; 120A(1): 63-71. PMid:12794694 http://dx.doi.org/10.1002/ajmg.a.10192

[6] Gramling R, Trask P, Nash J, et al. Family physicians' beliefs about genetic testing. Fam Med. 2004; 36(10): 691-692. PMid:15531978

[7] Doksum T, Bernhardt BA, Holtzman NA. Does knowledge about the genetics of breast cancer differ between nongeneticist physicians who do or do not discuss or order BRCA testing? Genet Med. 2003; 5(2): 99-105. PMID: 12644779. http://dx.doi.org/10.1097/01.GIM.0000055198.63593.32

[8] Wideroff L, Freedman AN, Olson L, et al. Physician use of genetic testing for cancer susceptibility: results of a national survey. Cancer Epidemiol Biomarkers Prev. 2003; 12(14): 295-303. PMID: 12644779.

[9] Stange KC, Fedirko T, Zyzanski SJ, et al. How do family physicians prioritize delivery of multiple preventive services? J Fam Pract. 1994; 38(3): 231-237. PMID: 8126402

[10] Kristiansen IS, Hjortdahl P. The general practitioner and laboratory utilization: why does it vary? Fam Pract. 1992; 9(1): 22-2, 7. PMID: 1634022. 
[11] Hill SL, Holtzman GL, Berry R, et al. The appropriate use of the duplex scan in carotid arterial disease. Am Surg. 1997; 63(8): 720-725. PMID: 9247441.

[12] Birbeck GL, Gifford DR, Song J, et al. Do malpractice concerns, payment mechanisms, and attitudes influence test-ordering decisions? Neurology. 2004; 62(1): 119-121. PMID: 14718712. http://dx.doi.org/10.1212/01.WNL.0000101709.87316.0C

[13] Bates DW, Kuperman GJ, Jha A, et al. Does the computerized display of charges affect inpatient ancillary test utilization? Arch Intern Med. 1997; 157(21): 2501-2508. PMID: 9385303. http://dx.doi.org/10.1001/archinte.1997.00440420135015

[14] Bushnell CD, Siddiqi Z, Goldstein LB. Improving patient selection for coagulopathy testing in the setting of acute ischemic stroke. Neurology. 2001; 57(7): 1333-1335. PMID: 11591863. http://dx.doi.org/10.1212/WNL.57.7.1333

[15] Stafford RS, Misra B. Variation in routine electrocardiogram use in academic primary care practice. Arch Intern Med. 2001; 161(19): 2351-2355. PMID: 11606151 http://dx.doi.org/10.1001/archinte.161.19.2351

[16] Hilsden RJ, McGregor E, Murray A, et al. Colorectal cancer screening: practices and attitudes of gastroenterologists, internists and surgeons. Can J Surg. 2005; 48(6): 434-440. PMID: 16417049.

[17] Winkenwerder W, Levy BD, Eisenberg JM, et al. Variation in physicians' decision-making thresholds in management of a sexually transmitted disease. J Gen Intern Med. 1993; 8(7): 369-373. PMID: 8410 http://dx.doi.org/10.1007/BF02600075

[18] Yuan H, Chung F, Wong D, et al. Current preoperative testing practices in ambulatory surgery are widely disparate: a survey of CAS members. Can J Anaesth. 2005; 52(7): 675-679. PMID: 16103378 http://dx.doi.org/10.1007/BF03016552

[19] McGillivray DL, Roberts-Brauer R, Kramer MS. Diagnostic test ordering in the evaluation of febrile children. Physician and environmental factors. Am J Dis Child. 1993; 147(8): 870-874. PMID: 8352221 http://dx.doi.org/10.1001/archpedi.1993.02160320072022

[20] Hicks RJ, Hamm RM, Bemben DA. Prostate cancer screening. What family physicians believe is best. Arch Fam Med. 1995; 4(4): 317-322. PMID: 7711917 http://dx.doi.org/10.1001/archfami.4.4.317

[21] Arar N SJAHPMNP. Providers' behavioral beliefs regarding the delivery of genomic medicine at the Veterans Health Administration. Per Med. 2010; 7(5): 485-494. http://dx.doi.org/10.2217/pme.10.47

[22] Cabana MD, Rand CS, Powe NR, et al. Why don't physicians follow clinical practice guidelines? A framework for improvement. JAMA. 1999; 282(15): 1458-1465. PMID: 10535437 http://dx.doi.org/10.1001/jama.282.15.1458

[23] Miles MB HA. Qualitative data analysis: an expanded sourcebook. 2nd ed. Thousand Oaks, CA: Sage Publications; 1994. 